Bangladesh Journal of Neuroscience 2016; Vol. 32 (1): 21-27

\title{
Effect of Mannitol on Serum Electrolytes in Stroke Patients
}

\author{
MIFTAUL JANNATH CHOWDHURY ${ }^{1}$, MD. ABU NAYEEM CHOWDHURY², CHOWDHURY MUHAMMAD \\ OMAR FARUQUE ${ }^{3}$, RASHED IMAM ZAHID ${ }^{4}$, SUPRAVADAS ${ }^{5}$, \\ MYTRAYEE DEV ROY ${ }^{6}$, HUSSAIN AHMED ${ }^{1}$
}

\begin{abstract}
:
Mannitol, an osmotic agent and a free radical scavenger with neuroprotective properties, have been reported in many studies to decrease cerebral oedema, infarct size and neurological deficit in patients with stroke. The present study was carried out in the Department of Pharmacology and Therapeutics in collaboration with the Departments of Medicine and Neurology of Sylhet M.A.G Osmani Medical College and Hospital to evaluate the effect of mannitol on serum electrolytes in stroke patients. A total number of Forty five (45) acute stroke patients were randomly allocated in two groups- 23 patients received mannitol and 22 patients did not receive mannitol. On Day 1 after measuring serum electrolyte levels of both groups, mannitol receiving group was given infusion mannitol 1 $\mathrm{gm} / \mathrm{kg} \mathrm{IV}$ bolus followed by $0.5 \mathrm{gm} / \mathrm{kg}$ IV 6 hourly till 3rd day and then again on Day 3 serum electrolyte levels of both groups were measured. Predisposing risk factors, egsmoking, hypertension, dyslipidaemia were also analyzed. RBS (Random Blood Sugar) and serum creatinine were estimated before treatment initiation with aim to assess hyperglycaemia and renal impairment respectively. Statistical analysis was done by SPSS program using unpaired 't' test between two groups and between Day 1 and Day 3 by paired ' $t$ ' test. The result showed that serum sodium and potassium at Day 1 and Day 3 were almost similar between mannitol receiving group and mannitol non-receiving group ( $P>0.05$ for each component). After infusion of mannitol till third day, no electrolyte imbalance was noted. The study depicts that mannitol can be safely given to stroke patients as there were no change in electrolyte balance till third day.
\end{abstract}

Key words: Stroke, Mannitol therapy, Serum electrolyte.

Introduction:

Stroke is a focal neurological deficit of acute onset whose symptoms may last for more than 24 hours $^{1}$ and which may lead either to recovery or physical disability or impairment of bodily functions or may culminate in death of the person ${ }^{2}$. It is most frequently caused by blockage of cerebral artery by embolus or atherosclerotic hemorrhagic plaque leading to insufficient oxygen supply to parts of the brain dependent on the patency of the artery ${ }^{3}$ (figure 1) or when a blood vessel in the brain bursts spilling blood into spaces surrounding brain cells (figure 2). Stroke is the third leading cause of death and probably the most important cause of long term disability in most western nations ${ }^{4}$, its incidence is progressing in Bangladesh as well ${ }^{5}$. Cerebral oedema is a major cause of early death and long term disability after stroke ${ }^{6}$. Cerebral injury have shown that there is a dense central core, surrounded by a less dense zone of penumbra. Neuronal death occurs in this central focus. On the other hand, cells in the zone of penumbra remain viable for upto several hours after stroke and may be salvaged by reperfusion or by neuroprotective agents to prevent further damage $^{7}$.

1. Asstt. Professor, Asstt. Professor, Department of Pharmacology and Therapeutics, Sylhet Women's Medical College.

2. Assot. Professor, Department of Pharmacology and Therapeutics, Sylhet Women's Medical College.

3. Medical Officer, Department of Cardiology, Sylhet M.A.G.Osmani Medical College Hospital

4. Dr. Rashed Imam Zahid, Registrar, Department of Neuromedicine, National Institute of Neurosciences and Hospital, Dhaka.

5. Dr. Suprava Das, Senior lecturer, Pharmacology unit, Faculty of medicine, AIMST University, Malaysia.

6. Dr. Mytrayee Dev Roy, Asstt. Professor, Department of Pharmacology and Therapeutics, Jalalabad Ragib Rabeya Medical College, Sylhet. 


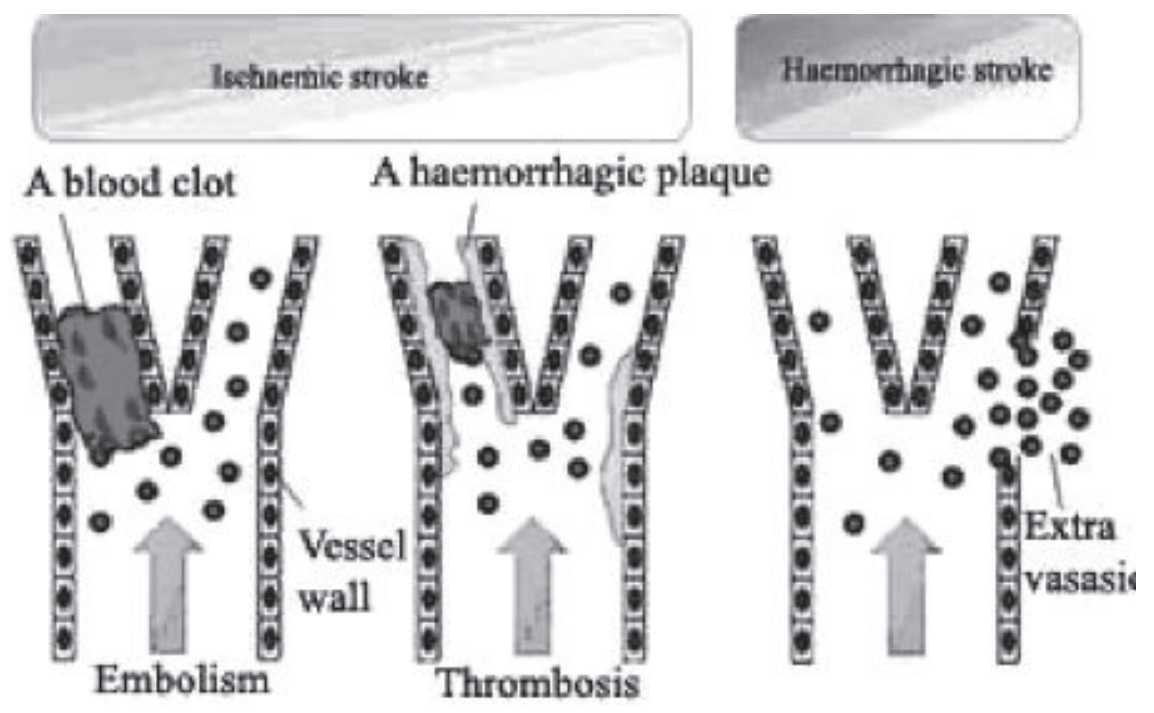

Fig.-1: Mechanisms of ischaemic and haemorrhagic strokes

Nerve cell injury most likely occurs from production of nitric oxide, changes in $\mathrm{Na} / \mathrm{K}$ gradients, release of glutamate and subsequent formation of free radicals ${ }^{7}$. CT scan appearance of ischaemic stroke is hypodensity in brain (figure 2) and that of haemorrhagic stroke is hyperdensity in brain (figure $3)$.

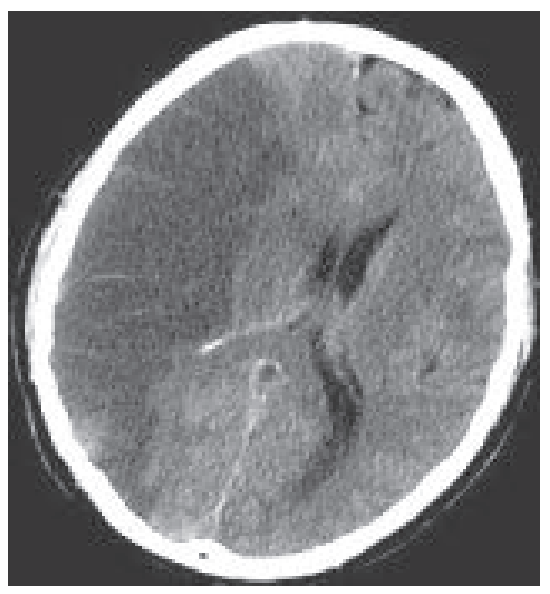

Fig.-2: CT scan slice of the brain showing a right hemispheric stroke (left side of image)

Classes of drugs under investigation for the treatment of acute stroke include those that promote early cerebral perfusion, neuroprotective agents and drugs to reduce cerebral oedema. Osmotic diuretics are among the agents that are widely used in the treatment of cerebral oedema ${ }^{8}$. Mannitol, an osmotic diuretic, decreases ICP (intracranial pressure), improves cerebral perfusion, acts as a

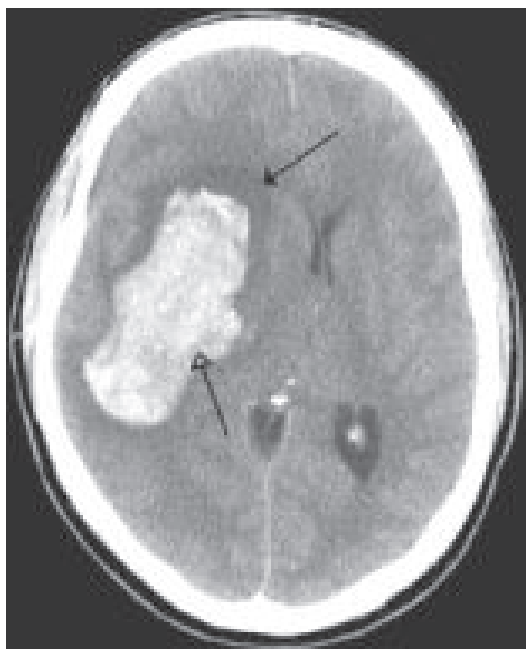

Fig.-3: An intraparenchymal bleed (bottom arrow) with surrounding oedema (top arrow)

free radical scavenger and also exerts neuroprotective effect. Mannitol decreases ICP by decreasing overall water content of the brain \& CSF (Cerebrospinal fluid) volume and by reducing blood volume due to vasoconstriction ${ }^{9}$. Mannitol also improves cerebral perfusion by decreasing viscosity and by improving red blood cell rheology 
(flow and shape of RBC), which results in increase brain oxygenation in large hemispheric stroke ${ }^{10}$. Most patients who survive the acute phase of a stroke regain some of the lost function ${ }^{11}$. The improvement of motor and sensory functions is accompanied by increased blood flow in impaired region surrounding the focal region ${ }^{12}$. Recovery after stroke is accelerated and facilitated by rehabilitation therapy, which might be supported by various drugs ${ }^{13}$. Therefore many trials were undertaken to enhance recovery of cognitive functions after stroke with the use of many pharmacological agents ${ }^{14}$ and have focused mainly those agents which improve cerebral reperfusion or neuroprotection ${ }^{15}$. The neuroprotective effect of mannitol thus takes place by reducing infarct size and number of apoptotic cells ${ }^{16}$. The neuroprotective effect of mannitol may also be due to scavenging of free radicals, which protects against biochemical injury. Mannitol has been used in human ischaemic brain damage for over 30 years ${ }^{17}$.

\section{Materials and Methods}

This interventional prospective comparative study was carried out during the period from 1st July, 2011 to 30th June, 2012 in the Department of Pharmacology and Therapeutics, in collaboration with the Departments of Medicine and Neurology of Sylhet M.A.G Osmani Medical College and Hospital. Newly diagnosed patients with stroke admitted into the Departments of Neurology and Medicine of this hospital within the study period were taken for the study. Patients with previous history of stroke, patients with serious co-morbid conditions, e.g.severe renal dysfunction, hepatic dysfunction, heart failure, malignancy, diabetes mellitus, diabetes insipidus, electrolyte imbalance, hyponatraemia, anaemia and hypovolaemic patients were excluded from the study. 73 patients were taken in the study. After fulfilling the inclusion and exclusion criteria, 45 acute stroke patients were selected for the study. History and clinical examination were recorded in a prescribed data collection form. Then patients were randomly allocated and categorized into two groups by all odd registration numbers in group-I and all even registration numbers in group-II. Patients who received mannitol belonged to group-É and who did not receive mannitol belonged to group-ÉÉ. Serum electrolyte levels of both groups were measured on Day 1 and Day 3. On Day 1 after measuring serum electrolyte level of both groups , group 1 was given infusion Mannitol $1 \mathrm{gm} / \mathrm{kg}$ I.V. bolus over $10-15$ minutes followed by $0.5-1 \mathrm{gm} / \mathrm{kg}$ I.V. 6 hourly ${ }^{18}$ till 3rd day and then again on Day 3 serum electrolyte level of both groups were measured. Other drugs, such as antiplatelet, anticoagulant, antihypertensive etc were given according to individual patient's need. Data from each patient were recorded in previously designed data collection sheet. Data were processed manually and analyzed with the help of SPSS (Statistical Package for Social Sciences) version 16.0. Then these parameters between two groups were compared. Comparison was done between mannitol receiving group and mannitol nonreceiving group by unpaired " $\mathrm{t}$ " test and between day 1 and day 3 by paired "t" test. A probability value $(p)$ of less than 0.05 was considered statistically significant.

\section{Results:}

For this study 73 (seventy three) stroke patients admitted in the Department of Medicine and Neurology of SOMCH were enlisted. Out of them, forty five (45) patients with acute stroke of first attack were selected during the study period from 1st July, 2011 to 30th June, 2012 after fulfilling the inclusion and exclusion criteria. 23 patients with acute stroke receiving mannitol at day 1 were enrolled as group-I and 22 patients with acute stroke without receiving mannitol were enrolled as group-II. Among the total stroke patients, the mean age was $62.6 \pm 11.6$ years. The mean age of patients of mannitol receiving group was $61.3 \pm 12.3$ years and mannitol non-receiving group was $64.0 \pm 11.0$ years respectively, which did not differ significantly $(t=-0.760 ; p=0.451)$ suggesting an age matched study.

Figure IV shows that among the total stroke patients, $35(77.8 \%)$ patients were male and $10(22.2 \%)$ patients were female. There were $17(73.9 \%)$ male and $6(26.1 \%)$ female patients in the mannitol receiving group; while $18(81.8 \%)$ male and 4 $(18.2 \%)$ female patients in mannitol non-receiving 


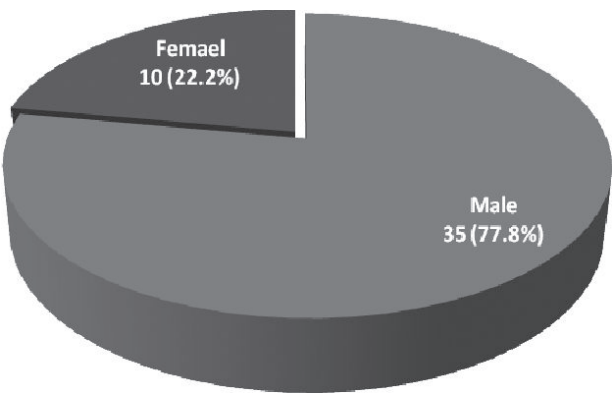

Fig.-4: Pie chart showing distribution of patients by $\operatorname{sex}(n=45)$

group. The sex difference between the two groups was not statistically significant $\left(\div^{2}=0.407 ; p=0.524\right)$ suggesting a sex matched study.

Table-I showed distribution of patients according to stroke type. It was observed that among the stroke patients, $34(75.6 \%)$ patients had haemorrhagic stroke and $11(24.4 \%)$ patients had ischaemic stroke. This table also showed that $18(78.3 \%)$ patients had haemorrhagic stroke and 5 (21.7\%) patients had ischaemic stroke in mannitol receiving group; while $16(72.7 \%)$ patients had haemorrhagic stroke and $6(27.3 \%)$ patients had ischaemic stroke in mannitol non-receiving group. The difference between the two groups was not statistically significant $\left(\dot{\leftarrow}^{2}=0.186 ; p=0.737\right)$.

Table-II showed the comparison of serum electrolytes between mannitol receiving group (Group-I) and mannitol non-receiving group (GroupII). It was observed that serum sodium in mannitol receiving group on Day 1 was $138.7 \pm 1.9$ and in non-receiving group was $139.0 \pm 3.1$, which was almost similar between two groups [ $t=-0.390$; $p=0.699]$. On Day 3, serum sodium was recorded as $140.1 \pm 2.7$ in mannitol receiving group and as $140.1 \pm 2.6$ in non-receiving group, which was almost similar between two groups $[\mathrm{t}=0.049$; $p=0.961]$. In mannitol receiving group serum sodium level at day 3 was significantly higher than that of day 1 [t=3.591; $p=0.002]$. In mannitol nonreceiving group serum sodium level at day 3 was also significantly higher than that of day $1[\mathrm{t}=2.483$; $p=0.022]$.

Serum potassium in mannitol receiving group on Day 1 was $4.1 \pm 0.5$ and in mannitol non-receiving group was $4.3 \pm 0.5$. It was almost similar between two groups [ $t=-0.994 ; p=0.326]$. On Day 3 serum potassium was recorded as $4.3 \pm 0.5$ in mannitol receiving group and as $4.5 \pm 0.5$ in mannitol nonreceiving group. It was almost similar between two groups [t=-0.910; $p=0.368]$. In mannitol receiving group serum potassium level at day 3 was significantly higher than that of day 1 [ $\mathrm{t}=2.750$; $p=0.012]$. In mannitol non-receiving group serum potassium level at day 3 was also significantly higher than that of day 1 [ $t=2.699 ; p=0.013]$.

On Day 1 serum chloride in mannitol receiving group was $102.9 \pm 3.7$ and in non-receiving group was $102.4 \pm 3.2$, which was almost similar between two groups [ $t=-0.249 ; p=0.804]$. On Day 3 serum chloride was recorded as $103.7 \pm 3.5$ in mannitol receiving group and as $103.2 \pm 2.8$ in non-receiving group, which was almost similar between two groups $[t=0.530 ; p=0.599]$. In mannitol receiving group serum chloride level at day 3 was significantly higher than that of day 1 [t=3.280; $p=0.003$ ]. But in mannitol non-receiving group serum chloride level was almost similar at day 3 and day 1 [ $t=1.178 ; p=0.252]$.

Table-I

Distribution of patients according to stroke type.

Study subjects

\begin{tabular}{lcccc}
\hline Stroke type & Group-I $(\mathrm{n}=234$ & Group-II & Total $(\mathrm{n}=45)$ & P value \\
\hline Haemorrhage & $18(78.3 \%)$ & $16(72.7 \%)$ & $34(75.6 \%)$ & \\
Ischaemic & $5(21.7 \%)$ & $6(27.3 \%)$ & $11(24.4 \%)$ & 0.737 \\
Total & $23(100.0 \%)$ & $22(100.0 \%)$ & $45(100.0 \%)$ & \\
\hline
\end{tabular}

Group-I: Mannitol receiving group of stroke patients.

Group-II: Mannitol non-receiving group of stroke patients. 


\section{Table-II}

Serum sodium, potassium and chloride levels of Group-I and Group-II patients at Day1 and Day 3. Data from mannitol receiving (targetted group) and non-receiving (control group) stroke patients.

\begin{tabular}{|c|c|c|c|c|c|c|c|c|c|}
\hline \multirow[t]{2}{*}{ Time } & \multicolumn{3}{|c|}{$\begin{array}{l}\text { Serum sodium }(\mathrm{mmol} / 1) \\
\text { Mean } \pm \mathrm{SD}\end{array}$} & \multicolumn{3}{|c|}{$\begin{array}{l}\text { Serum potassium }(\mathrm{mmol} / 1) \\
\text { Mean } \pm \mathrm{SD}\end{array}$} & \multicolumn{3}{|c|}{$\begin{array}{c}\text { Serum Chloride }(\mathrm{mmol} / 1) \\
\text { Mean } \pm \mathrm{SD}\end{array}$} \\
\hline & $\begin{array}{l}\text { Group-I } \\
(n=23)\end{array}$ & $\begin{array}{c}\text { Group-II } \\
(n=22)\end{array}$ & $p$ value & $\begin{array}{l}\text { Group-I } \\
(n=23)\end{array}$ & $\begin{array}{l}\text { Group-II } \\
(n=22)\end{array}$ & $P$ value & $\begin{array}{l}\text { Group-I } \\
(n=23)\end{array}$ & $\begin{array}{c}\text { Group-II } \\
(n=22)\end{array}$ & $p$ value \\
\hline Day I & $138.7 \pm 1.9$ & $139.0 \pm 3.1$ & ${ }^{*} 0.699$ & $4.1 \pm 0.5$ & $4.3 \pm 0.5$ & ${ }^{*} 0.326$ & $102.9 \pm 3.7$ & $102.4 \pm 3.2$ & ${ }^{*} 0.804$ \\
\hline Day 3 & $140.1 \pm 2.7$ & $140.1 \pm 2.6$ & ${ }^{\circ} 0.961$ & $4.3 \pm 0.5$ & $4.5 \pm 0.5$ & ${ }^{\circ} 0.368$ & $103.7 \pm 3.5$ & $103.2 \pm 2.8$ & ${ }^{\circ} 0.599$ \\
\hline Pvalue & ${ }^{*} 0.002$ & ${ }^{*} 0.022$ & & $\cdot 0.012$ & $\cdot 0.013$ & & $\Delta 0.003$ & ${ }^{\triangle} 0.252$ & \\
\hline
\end{tabular}

Group-I: Mannitol receiving group of stroke patients.

Group-II: Mannitol non-receiving group of stroke patients.

* = No significant difference in sodium, potassium and chloride between mannitol receiving group of stroke patients and non-receiving group of stroke patients on Day 1 at $p>0.05$ (Unpaired 't' test)

${ }^{\circ}=$ No significant difference in sodium, potassium and chloride between mannitol receiving group of stroke patients and non-receiving group of stroke patients on Day 3 at $p>0.05$ (Unpaired 't' test)

$\dagger=$ Significant difference in sodium in mannitol receiving group of stroke patients and non-receiving group of stroke patients on day 1 and Day 3 at $p=0.002$ and $p=0.022$ respectively (Paired ' $t$ ' test)

- = Significant difference in potassium in mannitol receiving group and non-receiving group on Day 1 and Day 3 at $p=0.012$ and $\mathrm{p}=0.013$ respectively( Paired ' $\mathrm{t}$ ' test)

$\Delta=$ Significant difference in chloride in mannitol receiving group on Day 1 and Day 3 at $p=0.003$ (Paired ' $t$ ' test )

$\triangle=$ No significant difference in chloride in mannitol non-receiving group on Day 1 and Day 3 at $p=0.252$ ( Paired ' $t$ ' test )

\section{Discussion:}

Mannitol is reported to decrease cerebral oedema, infarct size and neurological deficit in several experimental models of stroke ${ }^{16,19}$, mostly when administered within six hours after stroke onset. A prospective, interventional, comparative study was done to see the effect of mannitol on serum electrolytes in stroke patients. It was observed that serum sodium ( $138.7 \pm 1.9$ vs $139.0 \pm 3.1 ; p=0.699$ ) and serum potassium $(4.1 \pm 0.5$ vs $4.3 \pm 0.5$; $p=0.326$ ) at Day 1 were almost similar between mannitol receiving group and non-receiving group.

At Day 3, serum sodium (140.1 \pm 2.7 vs $140.1 \pm 2.6$; $\mathrm{p}=0.961)$ and serum potassium ( $4.3 \pm 0.5$ vs $4.5 \pm 0.5$; $p=0.368$ ) were also almost similar between mannitol receiving and non-receiving groups.

In this observation, significant variation was seen in the level of sodium and potassium levels between 1st day before infusion of mannitol (Day 1) and on 3rd day after infusion of mannitol (Day 3). In mannitol receiving group serum sodium and potassium levels at Day 3 were significantly higher than that of Day $1(140.1 \pm 2.7$ vs $138.7 \pm 1.9$; $p=0.002$ and $4.3 \pm 0.5$ vs $4.1 \pm 0.5 ; p=0.012$ respectively).

In mannitol non-receiving group serum sodium and potassium level on Day 3 was also significantly higher than that of Day $1(140.1 \pm 2.6$ vs $139.0 \pm 3.1$; $p=0.022$ and $4.5 \pm 0.5$ vs $4.3 \pm 0.5 ; p=0.013$ respectively).

These findings were supported by Rautaray ${ }^{18}$. They have done an observational study in MGM Medical College, Indore, India and found that mean values of sodium and potassium before giving mannitol (Day 1) were $130.4 \mathrm{meq} / \mathrm{l}$ and $3.9 \mathrm{meq} / \mathrm{l}$ respectively. After 3rd day of giving mannitol (Day 3 ) the mean values were $138.1 \mathrm{meq} / \mathrm{l}$ and $4.36 \mathrm{meq} /$ I for sodium and potassium respectively. In their study, statistically significant values for serum sodium $(P<0.001)$ and potassium $(P<0.02)$ were obtained when comparison was done between Day 1 and Day 3.

Current study revealed that infusion of mannitol $1 \mathrm{gm} / \mathrm{kg}$ stat and then $0.5 \mathrm{gm} / \mathrm{kg} 6$ hourly till $3 \mathrm{rd}$ day was sufficient to improve the clinical condition in stroke patients. The findings were in close 
conformity to the previously reported results that lower dosage of mannitol is quite effective with less chance of inducing hyperosmolar problems that have been noted with frequent high dose therapy ${ }^{20}$.

Regarding age and sex there were no significant difference between two groups, suggesting an age and sex matched study. The mean \pm SD age of total patients was $62.6 \pm 11.6$ years. Peak age of occurrence of stroke was $61-70$ years $(42.2 \%)$ and the males were predominant which is consistent with the findings of $\mathrm{YaO}^{20}$. They have analyzed 1027 patients with acute stroke. The average age of the study population was found to be 67.5 years and peak age of occurance of stroke was 59.5 73.2 years and the majority of stroke patients were male $(60.5 \%)$. In this study, number of ischaemic stroke and haemorrhagic stroke was almost similar between two groups. There was no significant difference in diagnosis between two groups $(P=0.737)$. The overall result indicates that mannitol can be successfully used in stroke patients, but further larger studies are needed to confirm the routine use of mannitol in stroke patients.

\section{Conclusion:}

A prospective interventional comparative study was conducted to evaluate the effect of mannitol on serum electrolytes in stroke patients. Forty five (45) patients of stroke were randomly allocated in two groups: 23 patients received mannitol and 22 patients did not receive mannitol. On Day 1 , after measuring serum electrolyte level of both groups, mannitol receiving group was given infusion mannitol $1 \mathrm{gm} / \mathrm{kg}$ IV bolus followed by $0.5 \mathrm{gm} / \mathrm{kg}$ IV 6 hourly till 3rd day and then again on Day 3 serum electrolyte levels of both groups were measured. It was observed that serum sodium, potassium and chloride on Day 1 and Day 3 were almost similar between mannitol receiving group and mannitol non receiving group ( $\mathrm{P}>0.05$ for each component). The study revealed that serum electrolyte levels rise within normal range and there was no adverse effect of mannitol infusion on serum electrolytes in stroke patients. Mannitol therefore may be safely given to patients of stroke as there was no electrolyte imbalance till third day. This study had some limitations. Firstly, the follow-up period of this study was only few days which might impede the total outcome of stroke. Secondly, sample size was small. Also there was lack of medical education in mass people. Despite the limitations and draw back we tried to do our work sincerely. The study needs further extensive investigation in large scale and for long term period. Further prospective interventional randomized trials and larger followup are needed for better assessment of long term efficacy and safety profile.

\section{References:}

1. Bamford, J .Clinical examination, diagnosis and sub-classification of stroke. Lancet 1992; 334: 400-4.

2. Roberts L, Councell C. Assessment of clinical outcome in acute stroke trials. Stroke 1998; 29: 986-91.

3. Noble S, Bendfield P .Piracetam: A review of its clinical potential in the management of patients with stroke CNS Drugs1998; 9(6): 497-511.

4. Bonita R Epidemiology of stroke Lancet 1992; 339(8789): 342-44.

5. Hannan M.A, Rahman M.M, Haque A, Ahmed H.U .Stroke: seasonal variation and association with hypertension. Bangladesh Med Res Counc Bull 2001; 27(2): 69-78.

6. Katzman R, Clasen R, Klatzo I. Report of joint committee for stroke resources: IV. Brain oedema in stroke. Stroke 1997; 8: 512-40.

7. Dalal P.M . Ischemic strokes: Management in first six hours Neurol India 2001; 49(2): 104-15.

8. James H.E. Methodology for the control of intracranial pressure with hypertonic mannitol. Acta Neurochirurgica 1980; 51: 161-72.

9. Davis M, Lucatorto M. Mannitol Revisited' Journal of Neuroscience Nursing 1994; 26 : 170-74.

10. Schwarz S, Schwab S, Bertram M. Effect of hypertonic saline hydroxyethyl starch solution and mannitol in patients with increased intracranial pressure after stroke. Stroke 1998; 29(8):1550-55. 
11. Kessler J, Thiel A, Karbe H. Piracetam improves activated blood flow and facilitates rehalitation of post stroke aphasic patients. Stroke 2000; 31(9): 2112-16.

12. Weiller C. Functional reorganization of the brain in recovery from striato-capsular information in man. Ann Neurol 1992; 31: 463-72.

13. Fissher M, Finklestein S. Pharmacological approaches to stroke recovery. Cerebrovascular Dis 1992; 9(5): 29-32.

14. Walker B.D .Pharmacotherapy in the treatment of aphasia. In: Goldstein 2B, ed. Restorative Neurology: Advances in pharmacotherapy for recovery after stroke. Armonk NY: Futura Publishing 1998; 157-270.

15. Orgogozo J.M. Piracetam in treatment of acute stroke. Pharmacopsychiat 1999; 32(1): 25-32.

16. Karibe $H$, Zarow G.J. Use of mild intra ischaemic hypothermia versus mannitol to reduce infarct size after temporary middle cerebral artery occlusion in rats. Journal of Neurosurgery 1995; 83: 98.

17. Bereczki D, Mihalka L, Szatmari S, Fekete $\mathrm{K}$, Di Casar D. Mannitol use in acute stroke: Case fetality at 30 days and 1 year. Stroke 2003; 34 (7): 1730-35.

18. Rautaray, S.S; Sharkar, P.D; Agrawal, B.K. Study of the effect of mannitol on serum electrolytes before and after three days in stroke patients. Stroke 2008, 19(3): 177 - 180.

19. Luvisotto T.L, Auer R.N, Sutherland G.R. The effect of mannitol on experimental cerebral ischemia. Neurosurgery 1996; 38(1): 13138.

20. Yao X.Y, Lin Y, Geng J.L, Sun Y.M. Age and gender specific prevalence of risk factors in patients with first ever ischaemic stroke in China. Stroke research and treatment 2012. [Art ID 136398. DOI:10.1155/2012/136398]. 